\title{
1Foreword to biophysical studies of membrane systems and interactions - 2 Commemorative issue in honour of Professor Michèle Auger
}

3 Dror E. Warschawski ${ }^{1}$, K.V. Lakshmi ${ }^{2}$ and Isabelle Marcotte ${ }^{3 *}$

4

$5^{1}$ Laboratoire des Biomolécules, LBM, CNRS UMR 7203, Sorbonne Université, École 6normale supérieure, PSL University, 75005 Paris, France

7

$8^{2}$ Department of Chemistry and Chemical Biology and The Baruch '60 Center for 9Biochemical Solar Energy Research, Rensselaer Polytechnic Institute, Troy, NY 12180, IOUSA

11

$12^{3}$ Department of Chemistry, Université du Québec à Montréal, P.O. Box 8888, Downtown 13Station, H3C 3P8, Montreal, Canada

14

15

$16 *$ Corresponding author

17Tel: 1-514-987-3000 \#5015

18Fax: 1-514-987-4054

19E-mail: marcotte.isabelle@uqam.ca

20

21

22 


\section{FOREWORD}

24 Professor Michèle Auger passed away in October 2018, after a long, fruitful and 25highly rewarding career in the field of membrane biophysics. Michèle was mostly 26interested in the importance of membranes as key biological interfaces, and in her 27research she deployed a wide range of experimental approaches to study them. She has 28contributed immensely to our knowledge on the physical chemistry of membranes, 29membrane interactions, and membrane-bound proteins and peptides through the 30application of solid-state nuclear magnetic resonance (SS-NMR), as well as infrared and 31Raman spectroscopy, and various other complementary techniques. This special issue, 32 which is dedicated to these topics, is a tribute to her career, with contributions by 33numerous collaborators and friends.

34 In consideration of Michèle's extensive contributions to the investigation of lipid 35bilayers, the enormous complexity of lipid systems and their role in peptide interactions 36are covered in this issue. In his review article, Hideo Akutsu nicely presents how 37 complementary spectroscopic approaches can allow one to better study the miscibility of 38various phospholipids in a bilayer [1]. The role of the phospholipid headgroups in 39creating interactions with membrane-active molecules is also illustrated in this review 40using antimicrobial peptides (AMPs), notably studied by Michèle together with her 41collaborators and students [2-4]. In their paper, Ramos et al. revisit the lamellar to 42 hexagonal phase transition in membranes as studied by ${ }^{31} \mathrm{P}$ and ${ }^{2} \mathrm{H}$ SS-NMR, calculating 43the partitioning constant of alkanes between, but also within, the different phases [5].

44 Membranes that are oriented in the magnetic field, either magnetically or 45mechanically, can bring valuable information on the position and interactions of proteins 46and peptides through SS-NMR studies. The development and application of such 47approaches have been amongst Michèle's many scientific contributions (for example [6489]). This special issue presents the characterization of new orientable systems and the 49application of oriented membranes to gather information on AMPs and lipids. A review 50 of two widely used lipid nano-objects - bicelles and nanodiscs - is presented by Erick 51Dufourc [10]. Moreover, in their paper, Park et al. explore the use of phospholipid 52polymer macrodiscs that align in the magnetic field as a new alternative to bicelles for 
53studying membrane proteins by SS-NMR [11]. Di Mauro et al. show that the insertion of 54paramagnetic tags in the outer polymer belt of lipid nanodiscs can reduce NMR 55acquisition times [12]. Gravel et al. propose new oriented membranes composed of 56phospholipids and Tween 80 - a detergent that is often used to isolate and stabilize 57 membrane proteins, thereby conveniently avoiding the detergent removal step prior to 58SS-NMR investigations [13]. They show that the orientation of these elongated vesicles 59can be flipped in the magnetic field when lanthanide ions are added, and they determine 60 the orientation of a model peptide. Michèle actively participated in this research together 61with her student Matthieu Fillion, and her former students Alexandre Arnold and Isabelle 62Marcotte.

63 In their paper, Jeong et al. tackle the structures of three promising AMPs by solution 64NMR, and their position relative to the membrane was studied by SS-NMR in bicelles 65[14]. While bicelles have originally been developed mostly for SS-NMR applications, 66Evans et al. expand on their use by performing electron paramagnetic resonance (EPR), 67 and circular dichroism (CD) spectroscopy studies, focusing on the transmembrane 68peptide fukutin-I [15]. The paper from Schmidt and Davis investigates liquid ordered69liquid disordered fluid phase coexistence in lipid mixtures [16]. Using two types of 70oriented membranes, i.e., magnetically (bicelles) and mechanically oriented bilayers, they 71 study the interaction of a synthetic peptide, and look at the lipid phase behavior as well as 72the orientation and dynamics of the peptide. Furthermore, Strandberg et al. explore the 73role of the positively charged residue at the N-terminus in the pore forming activity of 74AMP-analogs using mechanically oriented bilayers and ${ }^{15} \mathrm{~N}$ SS-NMR, as well as CD 75spectroscopy [17]. Piscidin 1, a biologically active host defense peptide originally 76obtained from fish, contains an unexpected amino terminal copper and nickel-binding 77 motif. Using a variety of biophysical methods, including ${ }^{15} \mathrm{~N}$ SS-NMR with oriented 78membranes, Paredes et al. show that metalation and oxidized-lipid incorporation 79improves its activity and that this peptide is more specific to anionic lipids [18].

80 Over the years, Michèle's work has been targeting important health issues such as 81antimicrobial resistance and amyloid-based diseases [19-21]. Recent work on the 82structure and membrane interactions of AMPs is presented in this special issue. Arias et 
83al. were able to identify the preferred location of individual fluorinated aromatic residues 84in various analogs of tritrpticin, either membrane-embedded or solvent-exposed, by 85solution ${ }^{19} \mathrm{~F}$ NMR methods [22]. Using an approach combining CD spectroscopy, with 86solution and SS-NMR, Sani et al. reveal that maculatin 1.1 adopts a transmembrane 87orientation in bilayers [23]. Raheem et al. study the mechanism of action of two aurein 882.2 analogs. Aurein has a strong antimicrobial effect against the human pathogen 89Staphylococcus aureus, and more active analogs can be generated from this peptide [24]. 90A paper from Paquet-Côté et al. involving both Michèle and her long-term collaborator 91and colleague Normand Voyer, studies the antimicrobial activity of crown-ether peptide 92analogs. It shows that increasing length improves the permeabilization activity of these 93crown peptides, and that peptides with larger crown ethers have increased 94permeabilization activity [25]. Using a novel in-cell approach, Santisteban et al. probe 95the interaction of two AMPs (MSI-78 and BP100) with the membranes of Gram(+) 96bacteria by ${ }^{2} \mathrm{H}$ SS-NMR [26].

97 Michèle also worked on a variety of proteins over the years, such as amyloid-forming 98peptides involved in Alzheimer's disease, the islet amyloid peptide from diabetes, a 99transglutaminase-inhibitor adduct, the photointermediates of bacteriorhodopsin and $\alpha$ 100 synuclein. Together with Robert Griffin, Ann McDermott, K.V. Lakshmi and others, she 101 was part of the revolution that brought SS-NMR to the fore as a major player for protein 102structure determination [27,28]. This special issue also presents investigations of other 103biologically-relevant peptides and proteins, such as ion channels, receptors and fiber104 forming proteins. In their paper, Aisenbrey and Bechinger approach the membrane 105interaction and topology, oligomerization and dynamics of the HIV gp41 protein, both $106 \mathrm{from}$ a lipid and from a protein perspective, through a variety of biophysical techniques 107including CD and fluorescence spectroscopy, as well as SS-NMR [29]. Ghosh and 108Welicky investigate the interaction of HIV gp41 and influenza HA fusion peptide by ${ }^{2} \mathrm{H}$ 109SS-NMR and reveal its insertion into the hydrophobic part of the bilayer [30]. Zhang and 110 McDermott study the effects of the membrane lipid composition on the functional states 111 of KcsA. Using SS-NMR, they show that anionic lipids would promote channel 112inactivation [31]. De Vlugt et al. employ through-bond SS-NMR spectroscopy to identify 113the flexible portions of the non-annular lipids that co-purify with the Anabaena Sensory 
114Rhodopsin (ASR) and find the presence of phosphatidyl ethanolamine lipids that are 115tightly bound to ASR [32]. Thompson and Baenziger review the effects of lipids on the 116function of pentameric ligand-gated ion channels [33]. Malik et al. investigate the 117differences in dynamics between the methylated and unmethylated states of a bacterial 118chemoreceptor using SS-NMR [34]. In their paper, Mark et al. use a sophisticated EPR 119experiment, called 2D HYSCORE, to determine the electronic structure of two redox120active tyrosine residues of photosystem II, which play an important role in water 121 oxidation driven by light energy [35]. Finally, Haya et al. investigate the effect of the 122pancreatic hormone glucagon on the lipid bilayer, and report morphological changes in 123DMPC bilayers as a function of the aggregation state of glucagon [36].

124 Michèle's spirit will continue to live within us when we get together to talk about our 125 science and in the scientific questions that we pursue in our laboratories. And, in 126acknowledging this, we can still feel her positive energy and her passion for science that 127she communicated to all of her students and scientific colleagues. We lost a wonderful 128and inspiring colleague and a dear friend far too early, but her memory will certainly stay 129 with us for a long time to come.

\section{0}

\section{ACKNOWLEDGEMENTS}

132We are indebted to Professor Hans Vogel for his constant help throughout the completion 133of this special issue of BBA-Biomembranes, in honour of Professor Michèle Auger.

\section{REFERENCES}

137

138[1] H. Akutsu, Structure and dynamics of phospholipids in membranes elucidated by 139combined use of NMR and vibrational spectroscopies, Biochim. Biophys. Acta, 140Biomembr., 1862 (2020) 183352.

141[2] M. Ouellet, J.-D. Doucet, N. Voyer, M. Auger, Membrane topology of a 14-mer 142model amphipathic peptide: A solid-state NMR spectroscopy study, Biochemistry, 46 143(2007) 6597-6606.

144[3] M. Fillion, M. Goudreault, N. Voyer, B. Bechinger, M. Auger, Amphiphilicity Is a 145Key Determinant in the Membrane Interactions of Synthetic 14-mer Cationic Peptide 146Analogues, Biochemistry, 55 (2016) 6919-6930. 
147[4] M. Bouchard, C. Paré, J.-P. Dutasta, J.-P. Chauvet, C. Gicquaud, M. Auger, 148Interaction between $\mathrm{G}$-actin and various types of liposomes: $\mathrm{A}{ }^{19} \mathrm{~F},{ }^{31} \mathrm{P}$ and ${ }^{2} \mathrm{H}$ nuclear 149magnetic resonance study, Biochemistry, 37 (1998) 3149-3155.

150[5] A.P. Ramos, M. Doroudgar, M. Lafleur, Determination of n-alkane partitioning 151 within phosphatidylethanolamine L $\alpha /$ HII phases, Biochim. Biophys. Acta, Biomembr., 1521862 (2020) 183201.

153[6] F. Picard, M.-J. Paquet, J. Levesque, A. Bélanger, M. Auger, ${ }^{31}$ P NMR first spectral 154moment study of the partial magnetic orientation of phospholipid membranes, Biophys. 155J., 77 (1999) 888-902.

156[7] I. Marcotte, M. Auger, Bicelles as model membranes for solid- and solution-state 157NMR studies of membrane peptides and proteins, Concepts Magn. Res., 24A (2005) 1715837.

159[8] I. Marcotte, A. Bélanger, M. Auger, The Orientation Effect of Gramicidin A on 160Bicelles and Eu3+-Doped Bicelles as Studied by Solid-State NMR and FTIR 161Spectroscopy, Chem. Phys. Lipids, 139 (2006) 137-149.

162[9] M. Fillion, M. Auger, Oriented samples: a tool for determining the membrane 163topology and the mechanism of action of cationic antimicrobial peptides by solid-state 164NMR, Biophys. Rev., 7 (2015) 311-320.

165[10] E.J. Dufourc, Bicelles and nanodiscs for biophysical chemistry, Biochim. Biophys. 166Acta, Biomembr., 1863 (2021) 183478.

167[11] S.H. Park, J. Wu, Y. Yao, C. Singh, Y. Tian, F.M. Marassi, S.J. Opella, Membrane 168proteins in magnetically aligned phospholipid polymer discs for solid-state NMR 169spectroscopy, Biochim. Biophys. Acta, Biomembr., 1862 (2020) 183333.

170[12] G.M. Di Mauro, N.Z. Hardin, A. Ramamoorthy, Lipid-nanodiscs formed by 171paramagnetic metal chelated polymer for fast NMR data acquisition, Biochim. Biophys. 172Acta, Biomembr., 1862 (2020) 183332.

173[13] A.E. Gravel, A.A. Arnold, M. Fillion, M. Auger, D.E. Warschawski, I. Marcotte, 174Magnetically-orientable Tween-based model membranes for NMR studies of proteins, 175Biochim. Biophys. Acta, Biomembr., 1862 (2020) 183379.

176[14] J.-H. Jeong, M. Kim, Y. Kim, NMR structural studies and mechanism of action of 177Lactophoricin analogs as antimicrobial peptides, Biochim. Biophys. Acta, Biomembr., 1781863 (2021) 183469.

179[15] L.S. Evans, R. Hussain, G. Siligardi, P.T.F. Williamson, Magnetically aligned 180membrane mimetics enabling comparable chiroptical and magnetic resonance 181spectroscopy studies, Biochim. Biophys. Acta, Biomembr., 1862 (2020) 183343. 182[16] M.L. Schmidt, J.H. Davis, ${ }^{2} \mathrm{H}$ NMR of oriented phospholipid/cholesterol bilayers 183containing an amphiphilic peptide, Biochim. Biophys. Acta, Biomembr., 1862 (2020) 184183196.

185[17] E. Strandberg, D. Bentz, P. Wadhwani, J. Bürck, A.S. Ulrich, Terminal charges 186modulate the pore forming activity of cationic amphipathic helices, Biochim. Biophys. 187Acta, Biomembr., 1862 (2020) 183243.

188[18] S.D. Paredes, S. Kim, M.T. Rooney, A.I. Greenwood, K. Hristova, M.L. Cotten, 189Enhancing the membrane activity of Piscidin 1 through peptide metallation and the 190presence of oxidized lipid species: Implications for the unification of host defense 191mechanisms at lipid membranes, Biochim. Biophys. Acta, Biomembr., 1862 (2020) 192183236. 
193[19] P.T. Lansbury, P.R. Costa, J.M. Griffiths, E.J. Simon, M. Auger, K.J. Halverson, 194D.A. Kocisko, Z.S. Hendsch, T.T. Ashburn, R.G.S. Spencer, B. Tidor, R.G. Griffin, 195Structural model for the beta-amyloid fibril based on interstrand alignment of an 196antiparallel-sheet comprising a C-terminal peptide, Nat. Struct. Biol., 2 (1995) 990-998. 197[20] I. Marcotte, K.L. Wegener, Y.-H. Lam, B.C.S. Chia, M.R.R. de Planque, J.H. 198Bowie, M. Auger, F. Separovic, Interaction of antimicrobial peptides fom Australian 199amphibians with lipid membranes, Chem. Phys. Lipids, 122 (2003) 107-120.

200[21] B. Martial, T. Lefèvre, M. Auger, Understanding amyloid fibril formation using 201protein fragments: structural investigations via vibrational spectroscopy and solid-state 202NMR, Biophys. Rev., 10 (2018) 1133-1149.

203[22] M. Arias, J.M. Aramini, N.D. Riopel, H.J. Vogel, Fluorine-19 NMR spectroscopy of 204fluorinated analogs of tritrpticin highlights a distinct role for Tyr residues in antimicrobial 205peptides, Biochim. Biophys. Acta, Biomembr., 1862 (2020) 183260.

206[23] M.-A. Sani, A.P. Le Brun, F. Separovic, The antimicrobial peptide maculatin self 207assembles in parallel to form a pore in phospholipid bilayers, Biochim. Biophys. Acta, 208Biomembr., 1862 (2020) 183204.

209[24] N. Raheem, P. Kumar, E. Lee, J.T.J. Cheng, R.E.W. Hancock, S.K. Straus, Insights 210 into the mechanism of action of two analogues of aurein 2.2, Biochim. Biophys. Acta, 211Biomembr., 1862 (2020) 183262.

212[25] P.-A. Paquet-Côté, J.-P. Paradis, M. Auger, N. Voyer, Crown ether modified 213peptides: Length and crown ring size impact on membrane interactions, Biochim. 214Biophys. Acta, Biomembr., 1862 (2020) 183261.

215[26] N.P. Santisteban, M.R. Morrow, V. Booth, Effect of AMPs MSI-78 and BP100 on 216the lipid acyl chains of ${ }^{2} \mathrm{H}$-labeled intact Gram positive bacteria, Biochim. Biophys. Acta, 217Biomembr., 1862 (2020) 183199.

218[27] R.G.S. Spencer, K.J. Halverson, M. Auger, A.E. McDermott, R.G. Griffin, P.T. 219Lansbury Jr., An unusual peptide conformation may precipitate amyloid formation in 220Alzheimer's disease: Application of solid-state NMR to the determination of protein 221 secondary structure, Biochemistry, 30 (1991) 10382-10387.

222[28] K.V. Lakshmi, M. Auger, J. Raap, J. Lugtenburg, R.G. Griffin, J. Herzfeld, 223Internuclear distance measurement in a reaction intermediate: Solid-state ${ }^{13} \mathrm{C}$ NMR 224rotational resonance determination of the Schiff base configuration in the $\mathrm{M}$ 225photointermediate of bacteriorhodopsin, J. Am. Chem. Soc., 115 (1993) 8515-8516. 226[29] C. Aisenbrey, B. Bechinger, Structure, interactions and membrane topology of HIV 227gp41 ectodomain sequences, Biochim. Biophys. Acta, Biomembr., 1862 (2020) 183274. 228[30] U. Ghosh, D.P. Weliky, ${ }^{2} \mathrm{H}$ nuclear magnetic resonance spectroscopy supports larger 229amplitude fast motion and interference with lipid chain ordering for membrane that 230 contains $\beta$ sheet human immunodeficiency virus gp41 fusion peptide or helical hairpin 231 influenza virus hemagglutinin fusion peptide at fusogenic $\mathrm{pH}$, Biochim. Biophys. Acta, 232Biomembr., 1862 (2020) 183404.

233[31] D. Zhang, G.S. Howarth, L.A. Parkin, A.E. McDermott, NMR studies of lipid 234regulation of the K+ channel KcsA, Biochim. Biophys. Acta, Biomembr., 1863 (2021) 235183491.

236[32] J.E. de Vlugt, P. Xiao, R. Munro, A. Charchoglyan, D. Brewer, M.S. Al-Abdul237Wahid, L.S. Brown, V. Ladizhansky, Identifying lipids tightly bound to an integral 238membrane protein, Biochim. Biophys. Acta, Biomembr., 1862 (2020) 183345. 
239[33] M.J. Thompson, J.E. Baenziger, Structural basis for the modulation of pentameric 240ligand-gated ion channel function by lipids, Biochim. Biophys. Acta, Biomembr., 1862 241(2020) 183304.

242[34] N. Malik, K.A. Wahlbeck, L.K. Thompson, Strategies for identifying dynamic 243regions in protein complexes: Flexibility changes accompany methylation in chemotaxis 244receptor signaling states, Biochim. Biophys. Acta, Biomembr., 1862 (2020) 183312. 245[35] B. Mark, C.S. Coates, S. Sugimura, A. Baldansuren, J. Ku, R.J. Debus, K.V. 246Lakshmi, Determining the Electronic Structure of Paramagnetic Intermediates in 247membrane proteins: A high-resolution 2D 1H hyperfine sublevel correlation study of the 248redox-active tyrosines of photosystem II, Biochim. Biophys. Acta - Biomembr., 1862 249(2020) 183422.

$250[36]$ K. Haya, Y. Makino, A. Kikuchi-Kinoshita, I. Kawamura, A. Naito, ${ }^{31} \mathrm{P}$ and ${ }^{13} \mathrm{C}$ 251solid-state NMR analysis of morphological changes of phospholipid bilayers containing 252glucagon during fibril formation of glucagon under neutral condition, Biochim. Biophys. 253Acta, Biomembr., 1862 (2020) 183290.

254 